\title{
A pilot study demonstrating cardiac uptake with 18F-florbetapir PET in AL
}

\section{amyloidosis patients with cardiac involvement}

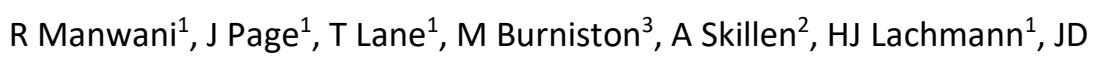

Gillmore $^{1}$, M Fontana ${ }^{1}$, C Whelan ${ }^{1}$, PN Hawkins ${ }^{1}$, T Wagner $^{2}$, AD Wechalekar ${ }^{1 *}$

${ }^{1}$ National Amyloidosis Centre, University College London (Royal Free Campus), Rowland Hill Street, London

${ }^{2}$ Department of Nuclear Medicine, Royal Free Hospital, Pond Street, London

${ }^{3}$ Department of Nuclear Medicine, Barts Health NHS Trust, West Smithfield, London

* Corresponding Author:

Dr Ashutosh Wechalekar, National Amyloidosis Centre, University College London (Royal Free Campus), Rowland Hill Street, London, UK, NW3 2PF

Telephone number: +44 207433 2733, Fax number: +44 2074332817

Email: a.wechalekar@ucl.ac.uk

Running head: 18F-florbetapir PET in cardiac amyloidosis

Abstract word count: 212

Text word count: 2707

Number of figures: 1

Number of tables: 4

Number of references: 16 


\section{$\underline{\text { Abstract }}$}

${ }^{18} \mathrm{~F}$-florbetapir is a promising tracer in amyloidosis. This study evaluates its use in patients with-systemic AL amyloidosis (AL) before and after treatment, as well as its serial utility in monitoring. Fifteen AL patients with cardiac involvement underwent ${ }^{18} \mathrm{~F}$-florbetapir PET imaging.-and $t$. Three underwent repeat imaging post-chemotherapy. hree patients underwent repeat imaging after chemotherapy.-All patients patients-had demonstrable cardiac uptake with ${ }^{18} \mathrm{~F}$-florbetapir. Cardiac uptake appeared greater in chemotherapynaïve vs chemotherapy-established AL patients median $f($ mean left ventricular retention index 0.21 vs $0.14 \mathrm{~min}^{-1}$, respectively) and greater in patients that had not achieved at least a partial haematological response (mean left ventricular retention index 0.2 vs $0.14 \mathrm{~min}^{-1}$, respectively). - There was no interval difference in cardiac uptake, and no correlation in cardiac uptake with cardiac biomarkers or serum free light chains. This is the largest study of ${ }^{18} \mathrm{~F}$-florbetapir in patients with-AL-amyloidosis. It is the first study-to include patients prior to-starting-before chemotherapy and uniquely includes patients who underwent repeat imaging after-post-chemotherapy. All patients had cardiac uptake with ${ }^{18} \mathrm{~F}$ florbetapir, regardless of haematological or NT-proBNP response to chemotherapy. There was a suggestion that treatment-naïve patients may have higher cardiac uptake. Larger studies are required to establish the role of this tracer in screening patients with a myloidosis for cardiac involvement, discriminating between ATTR and AL amyloidosis, and in disease monitoring.

Keywords: Amyloidosis; Florbetapir; PET; AL; imaging 
Abbreviations

123I-SAP: ${ }^{123}$ |-labeled serum amyloid P component

AL: systemic immunoglobulin light chain amyloidosis

ATTR: transthyretin amyloidosis

CR: complete haematological response

ECV: extracellular volume

MRI: magnetic resonance imaging

PET: positron emission tomography

NT-proBNP: N-terminal pro b-type natriuretic peptide

PR: partial haematological response

$\underline{\text { Rl: retention index }}$

SUV: standardised uptake value

VGPR: very good partial haematological response
Formatted: Font: Bold, Underline

Formatted: Font: +Body (Calibri), 12 pt, Font color: Text 1

Formatted: Font: Not Bold, No underline 


\section{Introduction}

The amyloidoses are a group of disorders characterised by extracellular deposition of amyloid fibrils, derived from pathological misfolding of precursor proteins. Cardiac involvement is a feature of both systemic immunoglobulin light chain (AL) and transthyretin (ATTR) amyloidosis, whereby the extracellular space of the heart is expanded by an amorphous fibrillary material, causing a restrictive cardiomyopathy. [1] Cardiac involvement is the critical determinant of outcomes in AL amyloidosis. Early diagnosis is key, as treatment delay in AL can lead to stark outcomes. Histological diagnosis is the gold standard but the risks of endomyocardial biopsy are not insignificant - especially in elderly, frail patients with other comorbidities.

Imaging has been a cornerstone of diagnosing cardiac amyloidosis. Echocardiography is the most widely used method but continues to lack specificity and sensitivity. [2] Cardiac magnetic resonance imaging (MRI) overcomes some of these limitations but is contraindicated in a substantial proportion of patients, and is only available in specialist centres with interpretation often dependent on the use of specific protocols. [3] ${ }^{123}$-labeled serum amyloid $\mathrm{P}$ component ( $\left.{ }^{123} \mathrm{I}-\mathrm{SAP}\right)$ scintigraphy binds specifically to amyloid deposits in viscera and is helpful to quantify and localise amyloid deposition, but is only available in two centres in the world and does not demonstrate cardiac amyloid deposits. [4] Bone seeking

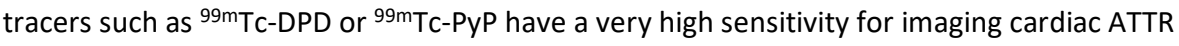
amyloidosis but are less sensitive in detecting other types of cardiac amyloidosis. [5] 
PET modalities have emerged in cardiac amyloidosis. Antoni et al reported increased cardiac uptake with ${ }^{11}$ Carbon-Pittsburgh compound $\mathrm{B}\left({ }^{11} \mathrm{C}-\mathrm{PIB}\right)$ in patients with ATTR and AL cardiac amyloidosis. [6] Lee et al reported a significant increase in ${ }^{11}$ C-PIB myocardial uptake in chemotherapy-naïve AL patients compared to already treated patients, but this tracer is only available in centres with a cyclotron. [7]

Dorbala's group performed a pilot study of PET imaging with ${ }^{18}$ F-florbetapir, a tracer with a high affinity for beta amyloid in the brain, in patients with ATTR and AL cardiac amyloidosis. [8] They found that left ventricular retention index (RI), target-to-background ratio, left ventricular myocardial standardised uptake values (SUV) and left ventricular myocardial to liver SUV were higher in patients with cardiac amyloidosis compared to controls. They also reported higher cardiac retention indices in patients with cardiac AL amyloidosis compared to patients with ATTR cardiac amyloidosis, although sample sizes were small and this finding was not statistically significant. Autoradiography studies have demonstrated ${ }^{18} \mathrm{~F}$-florbetapir uptake in autopsy cases of cardiac AL and ATTR amyloidosis, but no uptake in control samples. [9] Osborne et al reported delayed myocardial washout with ${ }^{18} \mathrm{~F}$-florbetapir imaging in 8 subjects with cardiac amyloidosis compared to 3 control subjects. [10] ${ }^{18} \mathrm{~F}$ florbetaben PET has shown similar promise in another study, differentiating between cardiac amyloidosis and hypertensive heart disease. [11] Uptake with 18F-florbetapir PET at extracardiac sites of amyloid deposition (spleen, tongue, parotids, fat, lungs and kidneys) has also been recently described. [12] 
Currently, clinical cardiac response to chemotherapy in patients with systemic $A L$ amyloidosis is defined by an improvement in NT-proBNP after chemotherapy. [13] This biomarker response has been found to predict long term survival, but other factors such as renal insufficiency, causes of heart failure independent of AL amyloidosis, immunomodulatory agents and acute heart failure can also lead to elevation of NT-proBNP and may not necessarily reflect true organ response. Echocardiographic and cardiac MRI imaging, whilst useful after treatment, are not used in routine evaluation of organ response.

In this pilot study, we evaluated cardiac uptake with ${ }^{18} \mathrm{~F}$-florbetapir PET in patients with systemic AL amyloidosis with cardiac involvement before and after treatment, as well as its serial utility in monitoring.

\section{Materials and methods}

All patients were recruited to a prospective study of ${ }^{18} \mathrm{~F}$-florbetapir in cardiac amyloidosis from September 2016 to June 2017. The inclusion criteria were: confirmed diagnosis of AL amyloidosis defined by amyloid deposition confirmed on a tissue biopsy and documented AL amyloid fibril typing, and evidence of cardiac involvement as defined by international amyloidosis consensus criteria. [14] The exclusion criteria were: inability to lie flat, NYHA Class IV heart failure, pregnancy or unwilling to undergo pregnancy test prior to study (in women of child bearing potential), the presence of any contraindication to undergoing PET imaging, and a known allergy to Amyvid. All patients were aged 40 years or over. 
The primary outcome was ${ }^{18} \mathrm{~F}$-florbetapir uptake in the heart. The variables of the study were left ventricular retention index (RI), mean and maximum left ventricular myocardial standardised uptake variable (SUV mean and SUV $\left.V_{\max }\right) .{ }^{18} \mathrm{~F}$-florbetapir RI was calculated by the method described by Dorbala et al, as the mean LV myocardial tissue radiotracer concentration between 10 and 30 minutes after injection of ${ }^{18} \mathrm{~F}$-florbetapir, divided by the integral of the blood pool ${ }^{18} \mathrm{~F}$-florbetapir time activity curve from 0 to 20 minutes. [8] A volume of interest in the left ventricular cavity was used by Dorbala's group. However, our group felt that counts in the left ventricular wall were spilling over into the blood pool volume of interest, potentially giving falsely low results in some patients. An approach was therefore taken using a volume of interest in the aorta for the blood pool instead, as this was less likely to include counts from other areas of uptake.

All patients underwent serial protocolized assessment at the UK National Amyloidosis Centre including full biochemical tests for organ function, serum free light chain measurement as well as serum and urine protein electrophoresis and immunofixation, cardiac biomarker measurement, echocardiography, cardiac MRI (unless contraindicated), and ${ }^{123}$ I-SAP scintigraphy. Organ involvement was defined as per international amyloidosis consensus criteria. [14]

\section{Imaging Protocol:}

Patients underwent list mode dynamic PET imaging for 60 minutes using a Siemens Biograph $\mathrm{PET} / \mathrm{CT}$ machine following a mean intravenous administration of $331 \mathrm{MBq}$ (range 294- 
$370 \mathrm{MBq})$ of ${ }^{18} \mathrm{~F}$-florbetapir. The heart was placed in the centre of the field of view. CT imaging was performed over this area using automatic exposure control (CARE Dose 4D, CARE kV - Siemens Healthcare) with exposure parameters of $65 \mathrm{mAs}$ and $120 \mathrm{kV}$. After dynamic imaging, the patient was asked to void. A half body acquisition PET-CT was then performed. Images underwent iterative reconstruction ( 2 iterations, 21 subsets) using time of flight information and point spread function modelling, with $2 \mathrm{~mm}$ Gaussian post filtering. The 60 minute list mode data was reconstructed into 37 frames (12 frames of $5 \mathrm{~s}$ each, 6 frames of $10 \mathrm{~s}$ each, 4 frames of $30 \mathrm{~s}$ each, 6 frames of $60 \mathrm{~s}$ each, 8 frames of $300 \mathrm{~s}$ each, and 1 frame of $600 \mathrm{~s})$.

Images were assessed by a nuclear medicine physician using HERMES workstation (HERMES Medical Solutions). The dynamic images (0-60 minutes) were assessed visually for cardiac uptake. The delayed half body images were visually analysed for uptake in the heart and other organs. All acquired data for all time frames was analysed and interpreted. For the dynamic images of the heart, the nuclear medicine physician assessed the presence of uptake in the myocardium and its localisation. For delayed half body images, cardiac uptake was assessed in the same manner. For extra-cardiac uptake, comparison was made with normal tracer distribution as described in the literature and as inferred from this study's patient population.

The study had approval from the institutional review board, and written consent was obtained from all patients in accordance with the Declaration of Helsinki. Statistical analysis was conducted using SPSS Version 24 software. 
Baseline characteristics are found in Table 1. Fifteen patients with systemic AL amyloidosis were included. Two patients underwent repeat imaging - before starting chemotherapy and after completion of treatment. All had cardiac involvement. Median age was 58 years (range 46-73 years). All patients had an ECOG score of 0-2 and the majority had NYHA class I-II symptoms. Median NT-proBNP was $2287 \mathrm{ng} / \mathrm{L}$ (range $114-6739 \mathrm{ng} / \mathrm{L}$ ). All patients had Mayo Stage III cardiac involvement at presentation. [15] Median ventricular wall thickness and left ventricular ejection fraction on echocardiography were $16 \mathrm{~mm}$ (range $13-18 \mathrm{~mm}$ ) and $54 \%$ (range $29-63 \%$ ), respectively.

Two patients were treatment-naïve at the time of the study, and two were early in their first cycle of chemotherapy (although one of the latter had achieved a haematological partial response at the time of imaging). At the time of imaging, the haematological response status was: patients in a complete haematological response (CR) - 9/12 (75\%), very good partial response (VGPR) - 2/12 (16.7\%), partial response (PR) - 1/12 (8.3\%). Of the three remaining patients, two were treatment-naïve and one was too early into chemotherapy for response assessment. Six patients had achieved a cardiac response as per international amyloidosis consensus criteria at the time of imaging. [14]

All patients had cardiac uptake with ${ }^{18} \mathrm{~F}$-florbetapir (Figure 1). There were no false negative scans. Primary outcome variables are presented in Table 2. The median LV retention index 
was $0.16 \mathrm{~min}^{-1}\left(0.05-0.29 \mathrm{~min}^{-1}\right)$. Median myocardial SUV $\max$ and $S U V_{\text {mean }}$ was 7.2 (range $0.8-15.7$ ) and 3.6 (range $0.8-7.1$ ), respectively. The pattern of uptake was diffuse. Uptake in the liver was seen in all cases as this tracer is excreted by the liver into the biliary system.

Previous reports suggested a lack of uptake in patients not "actively depositing" amyloid protein i.e. those in a haematological response. In this cohort, all patients in a clonal response still showed cardiac uptake. There was a suggestion that there was greater cardiac uptake in the treatment-naïve patients, although the number of treatment-naïve patients was too small for meaningful statistical analysis of difference in cardiac uptake between the two groups (Table 3). Patients that had not achieved at least a partial haematological response appeared to have higher LV retention indices, myocardial SUV $V_{\text {mean }}$ and myocardial SUV $V_{\max }$, although these findings were not statistically significant (Table 3). There was no statistically significant correlation with primary outcome variables and achievement of a cardiac response by NT-proBNP criteria. [13]

There was no statistically significant correlation between the retention indices of ${ }^{18} \mathrm{~F}$ florbetapir with global longitudinal strain rate, left ventricular ejection fraction, mean ventricular thickness, myocardial extracellular volume fraction (ECV) on cardiac MRI, serum troponin-T, serum NT-proBNP, difference in involved and uninvolved light chains ( $d F L C)$, and kappa:lambda ratio. 


\section{Comparison between initial and repeated ${ }^{18} \mathrm{~F}$-florbetapir imaging}

Three patients with AL had repeated ${ }^{18} \mathrm{~F}$-florbetapir imaging. At the time of initial imaging: one patient was treatment-naïve; one was early into first cycle of chemotherapy (but had achieved a haematological PR); one was on chemotherapy and in a haematological CR. All three patients were in a haematological CR at the time of repeat imaging and two had achieved an NT-proBNP response. There was no significant difference in primary outcome variables between initial and repeated imaging (Table 4).

\section{Discussion}

This is the largest study to date reporting on the use of ${ }^{18} \mathrm{~F}$-florbetapir PET imaging in AL patients with cardiac involvement. It is the first study to include patients that underwent ${ }^{18} \mathrm{~F}$-florbetapir imaging prior to starting chemotherapy, and the first to include repeat ${ }^{18} \mathrm{~F}$ florbetapir PET imaging at later timepoints. Crucially, this study also captures patients in differing haematological responses. It has previously been suggested that patients with normal light chains may have "inactive" amyloid deposits, which may not be associated with cardiac uptake with ${ }^{18} \mathrm{~F}$-florbetapir. There was a suggestion of greater cardiac uptake in patients with higher light chains than those in a good light chain response. However, all patients in this cohort had demonstrable cardiac uptake, irrespective of serum free light chain burden or NT-proBNP response. This is an important novel observation that offers new insight into the argument of 'active vs. 'inactive' amyloid hearts. 
Two patients in this study underwent ${ }^{18} \mathrm{~F}$-florbetapir imaging before starting chemotherapy. These patients appeared to have increased cardiac uptake compared to the rest of the cohort. Similarly, the three patients in this cohort who had not achieved at least a partial haematological response (two treatment-naïve patients and one in the first week of chemotherapy) appeared to have increased cardiac uptake, compared to those who did not. It must be emphasised that the latter two findings were not statistically significant. There remains a distinct possibility, however, that patients with particularly active clonal disease may have a higher cardiac disease burden, manifesting in increased cardiac uptake - but this relationship needs to be explored further in a study with larger patient numbers. Differing cardiac uptake in 'active' and 'inactive' amyloid deposits would have a potential role in monitoring treatment response as currently cardiac organ response after chemotherapy is defined as a $30 \%$ fall in NT-proBNP [13], but there are drawbacks of solely using a biomarker in assessment of cardiac response, when this biomarker is confounded by many other factors (e.g. renal insufficiency, immunomodulatory agents, acute fluid status and other causes of heart failure).

Three patients underwent follow-up imaging. At the time of initial imaging, one of these patients was treatment-naïve and subsequently went on to have chemotherapy, one patient had recently started chemotherapy and one had achieved a complete haematological response with chemotherapy. All patients were in a complete haematological response at the time of repeat imaging. There was no significant difference in cardiac uptake on initial and repeated imaging. However, the interval between imaging was short (range 2-8 
months) and future studies need to include greater numbers of patients and assess further the optimal timing of repeat ${ }^{18} \mathrm{~F}$-florbetapir imaging post-treatment.

No correlation was identified between primary outcome variables and cardiac biomarkers, LV ejection fraction, ventricular strain, ventricular wall thickness and ECV on cardiac MRI. The reasons for this are uncertain, but possibilities include a "saturation" effect due to a very high tracer affinity for amyloid, the heterogeneity of patients included in this cohort, or the relatively small sample size. Whist the uptake in all cases suggests a role for this tracer in diagnosis of cardiac amyloidosis, the lack of correlation with other markers may limit the utility of this tracer as a marker of prognosis in patients with more advanced cardiac amyloidosis.

The pattern of cardiac uptake in this study was diffuse and not restricted to the ventricular base as seen in ${ }^{99 \mathrm{~m} T c-D P D}$ or ${ }^{99 \mathrm{~m}} \mathrm{Tc}-\mathrm{PyP}$ imaging. The exact reason for this discrepancy is unclear but is likely to be due to the different mechanisms of tracer binding to amyloid deposits. ${ }^{99 \mathrm{~m}} \mathrm{Tc}$-DPD or ${ }^{99 \mathrm{~m}} \mathrm{Tc}$-PyP probably bind to calcium in amyloid deposits, whereas ${ }^{18} \mathrm{~F}$-florbetapir is fibril-specific. On endomyocardial biopsies, amyloid deposition can be seen throughout the myocardium and hence, we believe that diffuse cardiac uptake with ${ }^{18} \mathrm{~F}$-florbetapir reflects total amyloid deposition at the time of imaging. 
The findings of this study need to be interpreted within its limitations. The overall number of patients in this study is small. As there were no control subjects included in the cohort, it was not possible to evaluate specificity of this tracer for cardiac amyloidosis. Interval imaging was only performed in three AL patients and was carried out at differing timepoints in patients - further studies are needed to assess cardiac uptake at standardised timepoints before and after chemotherapy. Additionally, the utility of this tracer in early cardiac amyloidosis has yet to be defined as all patients in this study had clearly identifiable cardiac involvement.

${ }^{18} \mathrm{~F}$-florbetapir is licensed for detecting brain beta amyloid deposits in Alzheimer's disease. During the numerous studies of patients with Alzheimer's disease, imaging was limited to the head only and no systemic imaging details are available. Dorbala et al reported the first use of ${ }^{18} \mathrm{~F}$-florbetapir in 9 patients with cardiac amyloidosis (5 AL, 4 ATTR) and 5 control subjects. [8] Increased myocardial tracer uptake was seen in all amyloid subjects and none of the controls.

The small number of total subjects in studies thus far limits definitive conclusions on sensitivity and specificity, but both appear promising and make ${ }^{18} \mathrm{~F}$-florbetapir potentially attractive in distinguishing cases of true cardiac amyloid from non-amyloid pathologies which can share some echocardiographic and cardiac MRI appearances with amyloidotic infiltration. This is the first tracer to consistently demonstrate myocardial uptake in cardiac AL amyloidosis. This, if confirmed in larger studies, will be a step change in our approach to the diagnosis of cardiac AL amyloidosis. 
The striking appeal of ${ }^{18} \mathrm{~F}$-florbetapir is in its seemingly high affinity for cardiac amyloid deposits, as demonstrated by our study and previous literature. $[8,9,16]$ The exact place for this tracer in the amyloid diagnostic paradigm remains unclear. Larger studies are needed to clarify if this tracer will complement or, indeed, supplant the current methods of imaging cardiac amyloidosis.

\section{Acknowledgements}

Doses of ${ }^{18} \mathrm{~F}$-florbetapir for this study were supplied free of charge by Avid Pharmaceuticals. We also wish to acknowledge our staff in nuclear medicine, cardiac imaging, genetics, histopathology and those involved in the clinical care of our patients.

\section{Conflict of interest disclosures}

No author declares disclosures relevant for the manuscript or any conflict of interest or potential conflict of interest. 


\section{References}

1. Falk RH, Alexander KM, Liao R, et al. AL (Light-Chain) Cardiac Amyloidosis: A Review of

Formatted: Line spacing: Double Diagnosis and Therapy. J Am Coll Cardiol. 2016 Sep 20;68(12):1323-41. doi: 10.1016/j.jacc.2016.06.053. PubMed PMID: 27634125; eng.

2. Klein AL, Hatle LK, Burstow DJ, et al. Doppler characterization of left ventricular diastolic function in cardiac amyloidosis. J Am Coll Cardiol. 1989 Apr;13(5):1017-26. PubMed PMID: 2647814; eng.

3. Maceira AM, Joshi J, Prasad SK, et al. Cardiovascular magnetic resonance in cardiac amyloidosis. Circulation. 2005 Jan 18;111(2):186-93. doi:

10.1161/01.cir.0000152819.97857.9d. PubMed PMID: 15630027; eng.

4. Hawkins PN, Lavender JP, Pepys MB. Evaluation of systemic amyloidosis by scintigraphy with 123I-labeled serum amyloid P component. N Engl J Med. 1990 Aug 23;323(8):508-13. doi: 10.1056/nejm199008233230803. PubMed PMID: 2377176; eng.

5. Perugini E, Guidalotti PL, Salvi F, et al. Noninvasive etiologic diagnosis of cardiac amyloidosis using 99mTc-3,3-diphosphono-1,2-propanodicarboxylic acid scintigraphy. J Am Coll Cardiol. 2005 Sep 20;46(6):1076-84. doi: 10.1016/j.jacc.2005.05.073. PubMed PMID: 16168294; eng.

6. Antoni G, Lubberink M, Estrada S, et al. In vivo visualization of amyloid deposits in the heart with 11C-PIB and PET. J Nucl Med. 2013 Feb;54(2):213-20. doi: 10.2967/jnumed.111.102053. PubMed PMID: 23238792; eng.

7. Lee SP, Lee ES, Choi H, et al. 11C-Pittsburgh B PET imaging in cardiac amyloidosis. JACC Cardiovasc Imaging. 2015 Jan;8(1):50-9. doi: 10.1016/j.jcmg.2014.09.018. PubMed PMID: 25499132; eng. 
8. Dorbala S, Vangala D, Semer J, et al. Imaging cardiac amyloidosis: a pilot study using (1)(8)F-florbetapir positron emission tomography. European journal of nuclear medicine and molecular imaging. 2014 Sep;41(9):1652-62. doi: 10.1007/s00259-014-2787-6. PubMed PMID: 24841414.

9. Park MA, Padera RF, Belanger A, et al. 18F-Florbetapir Binds Specifically to Myocardial Light Chain and Transthyretin Amyloid Deposits: Autoradiography Study. Circ Cardiovasc Imaging. 2015 Aug;8(8). doi: 10.1161/circimaging.114.002954. PubMed PMID: 26259579; PubMed Central PMCID: PMCPMC4535193. eng.

10. Osborne DR, Acuff SN, Stuckey A, et al. A Routine PET/CT Protocol with Streamlined Calculations for Assessing Cardiac Amyloidosis Using (18)F-Florbetapir. Frontiers in Cardiovascular Medicine. 2015 05/08

01/21/received

04/23/accepted;2:23. doi: 10.3389/fcvm.2015.00023. PubMed PMID: PMC4671357.

11. Law WP, Wang WY, Moore PT, et al. Cardiac Amyloid Imaging with 18F-Florbetaben PET: A Pilot Study. J Nucl Med. 2016 Nov;57(11):1733-1739. doi: 10.2967/jnumed.115.169870. PubMed PMID: 27307344; eng.

12. Wagner T, Page J, Burniston M, et al. Extracardiac (18)F-florbetapir imaging in patients with systemic amyloidosis: more than hearts and minds. Eur J Nucl Med Mol Imaging. 2018 Jul;45(7):1129-1138. doi: 10.1007/s00259-018-3995-2. PubMed PMID: 29651545; PubMed Central PMCID: PMCPMC5953997. eng.

13. Comenzo RL, Reece D, Palladini G, et al. Consensus guidelines for the conduct and reporting of clinical trials in systemic light-chain amyloidosis. Leukemia. 2012 Nov;26(11):2317-25. doi: 10.1038/leu.2012.100. PubMed PMID: 22475872; eng.

14. Gertz MA, Comenzo R, Falk RH, et al. Definition of organ involvement and treatment response in immunoglobulin light chain amyloidosis $(\mathrm{AL})$ : a consensus opinion from the 
10th International Symposium on Amyloid and Amyloidosis, Tours, France, 18-22 April 2004. Am J Hematol. 2005 Aug;79(4):319-28. doi: 10.1002/ajh.20381. PubMed PMID: 16044444; eng

15. Dispenzieri A, Gertz MA, Kyle RA, et al. Serum cardiac troponins and N-terminal pro-brain natriuretic peptide: a staging system for primary systemic amyloidosis. J Clin Oncol. 2004 Sep 15;22(18):3751-7. doi: 10.1200/jco.2004.03.029. PubMed PMID: 15365071; eng

16. Wells K, Osborne D, Stuckey A, et al. 18F Florbetapir PET/CT cardiac amyloid imaging in patients with systemic amyloidosis. J Nucl Med. 2013 May 1, 2013;54(supplement 2):294. 
Table 1: Baseline characteristics.

\begin{tabular}{|l|l|}
\hline Overall cohort ( $\mathrm{n}=15)$ & Median (range) / $\mathrm{n}(\%)$ \\
\hline Age & $58(46-73)$ \\
\hline Male & $11(73 \%)$ \\
Female & $4(24 \%)$ \\
\hline ECOG: & \\
$0-2$ & $15(100 \%)$ \\
$3-4$ & 0 \\
\hline NYHA: & \\
I-II & $15(87 \%)$ \\
III-IV & $2(13 \%)$ \\
\hline Median NT-proBNP (ng/L) & $2287(114-6739)$ \\
\hline Median troponin T (ng/L) & $59(35-259)$ \\
\hline Median LV wall thickness (mm) & $16(13-18)$ \\
\hline Median LV ejection fraction (\%) & $54(29-63)$ \\
\hline Median 6 minute walk test (metres) & $472(138-662)$ \\
\hline Median serum creatinine (umol/L) & $114(66-203)$ \\
\hline Median 24 hour urinary protein (g/24h) & $0.3(0.1-7.2)$ \\
\hline
\end{tabular}




\begin{tabular}{|l|l|}
\hline Median serum albumin (g/L) & $\mathbf{4 2}(\mathbf{2 2 - 4 9 )}$ \\
\hline Median bilirubin (umol/L) & $\mathbf{8 ( 5 - 3 8 )}$ \\
\hline Median ALP (ULN 129 units/L) & $\mathbf{1 0 6}(\mathbf{4 5 - 1 7 0 2 )}$ \\
\hline Organ involvement: & \\
Cardiac & $15(100 \%)$ \\
Renal & $8(53 \%)$ \\
Liver & $4(24 \%)$ \\
Autonomic nerve & $2(12 \%)$ \\
Peripheral nerve & $1(6 \%)$ \\
Soft tissue & $4(24 \%)$ \\
Gastrointestinal & 0 \\
\hline Cardiac Mayo stage at presentation : & \\
I & 0 \\
II & 0 \\
III & $15(100 \%)$ \\
\hline Haematological response at time of & \\
imaging: & \\
CR & $9(60 \%)$ \\
VGPR & $2(13 \%)$ \\
PR & $1(7 \%)$ \\
No haematological response & $3(20 \%)$ \\
\hline NT-proBNP response at time of imaging & $6(40 \%)$ \\
No NT-proBNP response at time of & $9(60 \%)$ \\
imaging & \\
\hline
\end{tabular}

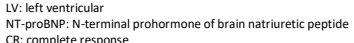

CR: complete response
Vopp: vever ood partial response
pep 
Table 2: Primary outcome variables.

\begin{tabular}{|c|c|c|c|c|c|c|c|c|c|c|c|c|c|c|c|}
\hline & 1 & 2 & 3 & 4 & 5 & 6 & 7 & 8 & 9 & 10 & 11 & 12 & 13 & 14 & 15 \\
\hline Diagnosis & $\mathrm{AL}$ & $\mathrm{AL}$ & $\mathrm{AL}$ & $\mathrm{AL}$ & $\mathrm{AL}$ & $\mathrm{AL}$ & $\mathrm{AL}$ & $\mathrm{AL}$ & $\mathrm{AL}$ & $\mathrm{AL}$ & $\mathrm{AL}$ & $\overline{\mathrm{AL}}$ & $\mathrm{AL}$ & $\overline{\mathrm{AL}}$ & $\mathrm{AL}$ \\
\hline $\begin{array}{l}\text { LV retention } \\
\text { index }\left(\min ^{-1}\right)\end{array}$ & 0.05 & 0.22 & 0.29 & 0.05 & 0.11 & 0.06 & 0.23 & No data & 0.11 & 0.19 & 0.17 & 0.25 & 0.22 & 0.14 & 0.13 \\
\hline $\begin{array}{l}\text { Myocardial } \\
\text { suV max }\end{array}$ & 1.6 & 6.5 & 12.4 & 0.8 & 2.1 & 5.8 & 15.7 & 5.4 & 7.2 & 7.2 & 9.9 & 9 & 9.4 & 7.2 & 7.9 \\
\hline $\begin{array}{l}\text { Myocardial } \\
\text { suV mean }\end{array}$ & 1.1 & 3.6 & 7.1 & 0.8 & 1.2 & 3.6 & 6.8 & 2.2 & 3.2 & 2.7 & 5.3 & 4.8 & 4.5 & 2.7 & 4.6 \\
\hline
\end{tabular}


Table 3: Relationship between primary outcome variables and clinical parameters.

\begin{tabular}{|c|c|c|c|c|c|c|}
\hline & \multicolumn{2}{|c|}{$\begin{array}{l}\text { LV retention index } \\
\left(\min ^{-1}\right)\end{array}$} & \multicolumn{2}{|c|}{ LV myocardial SUV $\max$} & \multicolumn{2}{|c|}{ LV myocardial SUV $V_{\text {mean }}$} \\
\hline $\begin{array}{l}\text { AL patients that were } \\
\text { treatment-naive }(n=2) \text {, } \\
\text { mean } \\
\text { AL patients established } \\
\text { on treatment }(n=13) \text {, } \\
\text { mean }\end{array}$ & \multicolumn{2}{|c|}{0.21 (range $0.17-0.25$ ) } & \multicolumn{2}{|c|}{9.45 (range $9-9.9$ ) } & \multicolumn{2}{|c|}{5.05 (range $4.8-5.3$ ) } \\
\hline $\begin{array}{l}\text { No NT-proBNP response } \\
(n=9) \text {, mean }\end{array}$ & $\begin{array}{l}0.19 \\
\text { (range } \\
0.11-0.29 \text { ) } \\
\\
0.16 \\
\text { (range } \\
0.05-0.25 \text { ) }\end{array}$ & $p=0.68$ & $\begin{array}{l}7.3 \\
\text { (range } \\
2.1-12.4 \text { ) } \\
\\
8.7 \\
\text { (range } \\
1.6-15.7 \text { ) }\end{array}$ & $p=0.52$ & $\begin{array}{l}3.9 \\
\text { (range } \\
1.2-5.2 \text { ) } \\
\\
4.3 \\
\text { (range } \\
1.1-6.8) \\
\end{array}$ & $p=0.73$ \\
\hline $\begin{array}{l}\text { Haematological partial } \\
\text { response (PR) or better } \\
\text { ( } n=12) \text {, mean }\end{array}$ & $\begin{array}{l}0.14 \\
\text { (range } \\
0.05-0.29 \text { ) } \\
\\
0.21 \\
\text { (range } \\
0.17-0.25 \text { ) }\end{array}$ & $p=0.11$ & $\begin{array}{l} \\
11.5 \\
\text { (range } \\
9-15.7) \\
\end{array}$ & $p=0.07$ & $\begin{array}{l} \\
5.63 \\
\text { (range } \\
4.8-6.8 \text { ) }\end{array}$ & $p=0.07$ \\
\hline
\end{tabular}


Table 4: Repeated ${ }^{18} \mathrm{~F}$-florbetapir imaging in three cardiac AL patients.

\begin{tabular}{|c|c|c|c|c|c|c|c|c|c|c|c|c|c|}
\hline & \multicolumn{6}{|c|}{ Initial 18f-florbetapir imaging } & \multicolumn{7}{|c|}{ Repeat ${ }^{18 F}$-florbetapir imaging } \\
\hline $\begin{array}{c}\text { Patient } \\
\text { S }\end{array}$ & $\begin{array}{l}\text { Treatment } \\
\text { Then }\end{array}$ & $\begin{array}{l}\text { Haematologic } \\
\text { response }\end{array}$ & $\begin{array}{l}\text { NT- } \\
\text { proBNP } \\
\text { response }\end{array}$ & $\begin{array}{l}\text { LV RI } \\
\left(\min ^{-1}\right)\end{array}$ & $\begin{array}{l}\text { SUV } \\
\max \end{array}$ & $\begin{array}{l}\text { SUV } \\
\text { mean }\end{array}$ & $\begin{array}{l}\text { Treatment } \\
\text { Then }\end{array}$ & $\begin{array}{l}\text { Interval } \\
\text { (months) }\end{array}$ & $\begin{array}{l}\text { Haematologic } \\
\text { response }\end{array}$ & $\begin{array}{l}\text { NT- } \\
\text { proBNP } \\
\text { response }\end{array}$ & $\begin{array}{l}\frac{\mathrm{LV} \mathrm{RI}}{\left(\mathrm{min}^{-1}\right)} \\
\end{array}$ & $\begin{array}{l}\text { SUV } \\
\max \end{array}$ & $\begin{array}{l}\text { SUV } \\
\text { mean }\end{array}$ \\
\hline 1 & $\begin{array}{l}\text { Day 15, cycle } 1 \\
\text { bortezomib, } \\
\text { cyclophosphamide, } \\
\text { dexamethasone }\end{array}$ & $\begin{array}{l}\text { Partial } \\
\text { response }\end{array}$ & No & 0.05 & 1.6 & 1.1 & $\begin{array}{l}7 \text { months post } \\
\text { chemotherapy }\end{array}$ & 2 & $\begin{array}{l}\text { Complete } \\
\text { response }\end{array}$ & Yes & 0.06 & 1.4 & 1.0 \\
\hline 3 & $\begin{array}{l}\text { Carfilizomib, } \\
\text { pomalidomide, } \\
\text { dexamethasone } \\
\text { cycle 11 }\end{array}$ & $\begin{array}{l}\text { Complete } \\
\text { response }\end{array}$ & Yes & 0.29 & 12.4 & 7.1 & $\begin{array}{l}\text { Carfilzomib, } \\
\text { pomalidomide, } \\
\text { dexamethasone } \\
\text { Cycle } 14\end{array}$ & 8 & $\begin{array}{l}\text { Complete } \\
\text { response }\end{array}$ & Yes & 0.19 & 11.8 & 4.7 \\
\hline 12 & Pre-chemotherapy & Not applicable & No & 0.25 & 9 & 4.8 & $\begin{array}{l}\text { Completed 2 } \\
\text { cycles of } \\
\text { chemotherapy }\end{array}$ & 8 & $\begin{array}{l}\text { Complete } \\
\text { response }\end{array}$ & No & 0.21 & 10.7 & 5.2 \\
\hline \multicolumn{11}{|c|}{ imary outcome } & $\mathrm{p}=0.3$ & $p=0.71$ & $p=0.5$ \\
\hline
\end{tabular}




\section{Figure 1. Myocardial uptake with ${ }^{18} \mathrm{~F}$-florbetapir was demonstrated in all patients in the}

study.

a) Diffuse, intense myocardial uptake is seen in a patient with cardiac AL amyloidosis undergoing first cycle of chemotherapy. b) Markedly intense myocardial uptake with ${ }^{18} \mathrm{~F}$ florbetapir in a patient with cardiac AL amyloidosis, prior to chemotherapy initiation. c) Diffuse, low grade myocardial uptake in a patient with cardiac AL amyloidosis in a complete clonal response three months after completion of chemotherapy. 
SSupplemental data: Clinical characteristics of entire cohort at the time of initial ${ }^{18} \mathrm{~F}$ -

florbetapir imaging 